\title{
Einführung TARMED im UV-/MV-/IV-Bereich per 1. Mai 2003
}

Herr

Dr. med. Hans Heinrich Brunner

Präsident FMH

Verbindung der Schweizer Ärzte

Elfenstrasse 18

3000 Bern 16

Zürich, 19. Dezember 2002

Sehr geehrter Herr Dr. Brunner

Lieber Hans Heiri

Der ZV hat beschlossen, den TARMED im UV-/ MV-/IV-Bereich am 1. Mai 2003 einzuführen. Solche Termine sollten unserer Meinung nach nicht einfach vom ZV verfügt, sondern nach Rücksprache mit den ausführenden Organen, hier den kantonalen Ärztegesellschaften, erlassen werden.

\section{Einführungsdauer und Schulung der Ärztinnen und Ärzte}

Wie wir Dir bereits in unserem Brief vom 23. August 2002 und erneut mündlich an der G7-Sitzung vom 12. Dezember 2002 mitgeteilt haben, brauchen wir im Kanton Zürich mindestens 6 Monate zur Einführung von TARMED (ab dem Datum, an welchem der definitive Einführungstermin mitgeteilt wird). Es kann gut sein, dass in kleineren Kantonen die Einführung innerhalb einer kürzeren Zeit vorgenommen werden kann. Es macht aber einen grossen Unterschied, ob in einem Kanton einige Dutzend oder rund 2500 Ärztinnen und Ärzte in die Handhabung des neues Tarifs eingeführt werden müssen. Für Zürich heisst dies, dass wir mindestens zwölf 4stündige Einführungsseminare mit kompetenten Referenten (Milizsystem) organisieren und durchfuhren müssen. Wir haben die Seminare für das 3. und 4. Quartal 2003 geplant. Die entsprechenden Schulungsunterlagen sind bereit. Eine Vorverschiebung der Schulungen ins 1. Quartal 2003 ist schlicht unmöglich.

\section{Software}

Wir haben uns bei den Ärztesoftwarefirmen über den aktuellen Stand der EDV in den Praxen erkundigt. Entsprechende Umfragen haben ergeben, dass bis jetzt erst etwa 50\% der Kolleginnen und Kollegen ihre Praxishardware und -software so modifiziert haben, dass eine Umstellung auf das TARMED-Programm möglich ist. Die lange andauernde und ständige «Verschieberei» des
TARMED-Einführungstermins hat leider dazu geführt, dass die verschiedenen Aufrufe zur Anpassung der Praxis-PCs von Seiten der FMH, der AGZ, der Fachgesellschaften und der Softwarefirmen nicht mehr ernst genommen - oder noch schlimmer - schon gar nicht mehr gelesen wurden. Allein im Kanton Zürich bedeutet das, dass über 1000 Praxen mit neuer, TARMED-tauglicher Hardund/oder Software ausgestattet werden müssen. Für Umrüstung in diesem Ausmass benötigen die Softwarefirmen mindestens 6 bis 12 Monate.

Einführung im Kanton Zürich per 1. Juli 2003 Wir müssen Dir mitteilen, dass die AGZ weder fähig noch willens ist, im Kanton Zürich den TARMED im UV-/MV-/IV-Bereich am 1. Mai 2003 einzuführen. Wir werden im Interesse des TARMED auch nicht zögern, bei einem so unrealistischen Einführungstermin (genau so, wie wir dies bei einem ungenügenden Starttaxpunktwert im KVG-Bereich tun werden) unsere Mitglieder aufzufordern, den Vertrag mit der MTK nicht zu unterschreiben. Der frühest mögliche Einführungstermin im Kanton Zürich wäre der 1. Juli 2003. Dies aber auch nur unter der Bedingung, dass Anfang Januar 2003 folgende Bedingungen erfüllt sind:

- der Einführungstermin vom 1. Juli 2003 wird anfangs Januar 2003 definitiv bestätigt;

- die Übergangsregelung für vital gefährdete Disziplinen ist unterzeichnet;

- die gesamten Daten/Informatik sind an die Vertragsparteien übergeben;

- das Abrechnungsformular ist definiert;

- die Probleme um den Validator sind gelöst;

- die Regelung über die Limitationen vor Einführung der elektronischen Abrechnung steht und ist den Ärzten mitgeteilt;

- der Starttaxpunktwert bleibt im UV-/MV-/IVBereich für AL und TL bei Fr. 1.-; 
- die den TARMED gefährdenden Interpretationsprobleme (Aktenstudium, Nachführen der Krankengeschichte, usw.) können gelöst werden.

Im übrigen halten wir daran fest, dass der optimale Einführungstermin für alle Versicherungsbereiche der 1. Januar 2004 ist. Mit einem solchen einheitlichen Einführungstermin kann die Schulung optimal gestaltet und die elektronische Umrüstung der Praxen bewältigt werden. Nachdem auch die Spitäler im UV-Bereich erst ab dem 1. Januar 2004 auf TARMED umstellen werden, wäre zur Not auch ein flexibler Einführungstermin für die Kantone denkbar. Mit diesem Vorgehen bestünde zumindest eine realistische Aussicht, dass die Umrüstung der Praxen zeitgerecht erfolgen kann. Gleichzeitig würden die Softwarefirmen etwas entlastet.

Nachdem jahrelang der Einführungstermin für TARMED immer wieder aus verschiedenen Gründen verschoben wurde, macht es unseres Erachtens wirklich keinen Sinn, den Tarif nur in einem Bereich in einer übereilten Aktion ein- führen zu wollen. Wenn die Ärztinnen und Ärzte nicht seriös vorbereitet und vor allem ihre Praxis-PCs umgerüstet sind, ist TARMED zum vornherein zum Scheitern verurteilt. Haben wir dafür über 15 Jahre lang viel Geld und unendlich viel Zeit investiert?

Wir hoffen sehr, dass der ZV auf seinen Beschluss zurückkommt, den geplanten Einführungstermin vom 1. Mai 2003 aufhebt und nach Rücksprache sowohl mit den kantonalen Ärztegesellschaften wie auch mit den Softwarefirmen einen realistischen neuen Termin festlegt.

Deiner baldigen Antwort sehen wir gerne entgegen und verbleiben mit kollegialen Grüssen.

Ärztegesellschaft des Kantons Zürich AGZ

Der Präsident:

Dr. med. Urs Stoffel

Der Leiter des Ressorts Sozialtarife: Dr. med. Jürg Pellaton

Dieses Schreiben geht per Mail auch an die Präsidenten der kantonalen Ärztegesellschaften.

\section{An die Präsidenten der Kantonalen Ärztegesellschaften}

Bern, 19. Dezember 2002

\section{Liebe Kollegen}

In Erwiderung des Briefes der AGZ halten wir folgende Punkte fest:

1. Der UV-/MV-/IV-Tarif ist ein schweizerischer Tarif, der national eingeführt wird, d. h. ohne Zustimmung der kantonalen Ärztegesellschaften oder Gesundheitsdepartemente.

2. Als Einführungstermin war der 1. Januar 2003 vorgesehen; wir konnten dieses Datum bis 1. Mai 2003 verschieben. Eine weitere Verschiebung würde zu Neuverhandlungen führen. Dies würde insbesondere auch Neuverhandlung des Starttaxpunktwertes bedeuten, der in der heutigen Situation kaum bei Fr. 1.- gehalten werden könnte. Über die Bedeutung dieses Starttaxpunktwertes für die kantonalen Verhandlungen müssen an diesem Ort keine weiteren Ausführungen gemacht werden.

3. Die von der AGZ wiederholten Forderungen für die Einführung des Tarifs sind erfüllt mit Ausnahme der Forderung Datum (selbstredend) und der sogenannten Validatoren. Wir halten in diesem Zusammenhang fest, dass für den Rechnungsverkehr diese Validatoren nicht zum Einsatz kommen, ohne dass zwischen FMH und Versicherern eine einvernehmliche Lösung vertraglich vereinbart ist, d.h. also nicht in der initialen Phase der Abrechnung nach neuem UV-/MV-/IV-Arzttarif.

4. Der Vertrag mit MTK, MV, IV ist seit 1. Januar 2002, damals noch vorbehältlich der zwischenzeitlich erfolgten Genehmigung in der Urabstimmung unterzeichnet. Ein weiteres Verschieben würde eine Vertragskündigung mit den obigen Folgen nach sich ziehen müssen.

5. Schulungen werden seit dem Jahr 2000 angeboten; die Versionen TARMED haben seit dieser Zeit keine wesentlichen Veränderungen mehr erfahren. Wenn Ärztinnen und Ärzte oder Gremien auf die Inanspruchnahme dieser Dienstleistungen verzichtet haben in der Annahme, TARMED werde ohnehin nie kommen, so kann dies nicht die Richtschnur für unser Vorgehen sein. 
6. Mutatis mutandis kann dies von den Softwarehäusern gesagt werden. Alle, die für die Einführung bereit stehenden Softwarehäuser, strafen diejenigen Lügen, die behaupten, sie hätten sich nicht vorbereiten können. Anzufügen wäre noch, dass in einer Vielzahl von Praxen nur wenige Rechnungen anfallen, die nach dem UV-/MV-/IV-Vertrag abgerechnet werden; die Arbeiten können in einer Übergangszeit sicher auch «von Hand» bewältigt werden.
7. Abschliessend halten wir fest, dass über das gesamte Procedere in Rundschreiben und/ oder Artikeln der Schweizerischen Ärztezeitung informiert wurde, so zuletzt an der vergangenen Präsidentenkonferenz, damals noch mit Einführungsdatum 1. April 2003.

Mit freundlichen Grüssen

Dr. H. H. Brunner

FMH-Präsident

\section{A l'attention des présidents des sociétés cantonales de médecine}

Berne, le 19 décembre 2002

Chers Confrères,

En réponse à la lettre ci-jointe de la Société de médecine du canton de Zurich, nous constatons les faits suivants:

1. Le tarif AA/AM/AI est un tarif suisse, introduit à l'échelon national sans l'approbation des sociétés cantonales de médecine ou des départements cantonaux de la santé publique.

2. La date d'introduction avait tout d'abord été fixée au $1^{\text {er }}$ janvier 2003. Nous sommes parvenus à repousser cette échéance jusqu'au $1^{\text {er }}$ mai 2003. Un nouvel ajournement demanderait de nouvelles négociations. Cellesci comprendraient notamment une renégociation de la valeur initiale du point tarifaire, qui, vu la situation actuelle, ne pourrait guère être maintenue à Fr. 1.-. Quant à impact de cette valeur initiale sur les négociations menées au niveau cantonal, il n'y a pas lieu de le commenter ici.

3. Les exigences formulées à plusieurs reprises par la société zurichoise sont satisfaites, sauf en ce qui concerne la date d'introduction (c'est évident) et les logiciels de contrôle. A cet égard, il convient de relever que ces «validateurs» ne seront pas utilisés pour la facturation tant que la FMH et les assureurs n'auront pas convenu, par contrat, d'une solution acceptable pour tous. En d'autres termes, il n'en sera pas fait usage pendant la phase initiale de facturation selon le nouveau tarif médical AA/AM/AI.

4. Une convention a été signée le $1^{\text {er }}$ janvier 2002 avec la CTM, l'AA et l'AI, sous réserve de son approbation lors de la votation générale, laquelle a eu lieu depuis. Un nouveau renvoi de l'introduction du tarif entraînerait une dénonciation de cette convention, suivie des conséquences évoquées ci-dessus.

5. Des formations ont été proposées dès l'an 2000, et les versions du TARMED développées depuis lors n'ont pas amené de nouveautés importantes. Si des médecins ou certains organes ont renoncé à faire usage de ces prestations dans l'idée que le TARMED ne verrait de toute façon jamais le jour, nous ne saurions tenir compte de cette attitude dans notre future marche à suivre.

6. La même remarque s'applique, à quelques nuances près, aux concepteurs de logiciels. Le fait que plusieurs d'entre eux soient prêts pour l'introduction du nouveau tarif apporte un démenti à l'affirmation selon laquelle il n'aurait pas été possible de se préparer. Il conviendrait encore de préciser que, dans beaucoup de cabinets médicaux, seul un petit nombre de factures sont établies en vertu de la convention portant sur le domaine AA/AM/AI. Nul doute que cette tâche pourrait aussi, pendant une période transitoire, être exécutée de façon manuelle.

7. Finalement, nous rappelons que des informations sur le sujet ont été diffusées par le biais de circulaires et/ou d'articles dans le Bulletin des médecins suisses. Le dernier rapport en date remonte à la Conférence des présidents du mois de novembre, alors que la date d'introduction du tarif était encore fixée au $1^{\text {er }}$ avril 2003.

Nous vous prions d'agréer, chers Confrères, nos salutations les meilleures.

Dr H. H. Brunner

Président de la FMH 